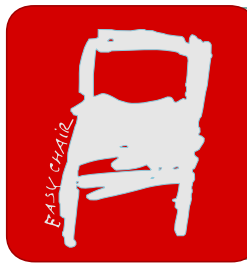

EPiC Series in Health Sciences

Volume 1, 2017, Pages 171-174

CAOS 2017. 17th Annual Meeting of the International Society for Computer Assisted Orthopaedic Surgery

\title{
Automation of a Flexoscope for Laser Osteotomy
}

\author{
Gabor Kosa ${ }^{1}$, Gregorio Aiello ${ }^{1}$, Azhar Zam², Philippe C. Cattin ${ }^{3}$, Georg \\ Rauter $^{1}$ \\ 1,* BIROMED-Lab, DBE, University of Basel, Allschwil, CH-4123, Switzerland, \\ ${ }^{2}$ BLOG, DBE, University of Basel, Allschwil, CH-4123, Switzerland \\ ${ }^{3}$ CIAN, DBE, University of Basel, Allschwil, CH-4123, Switzerland \\ (gabor.kosa, gregorio.aiello, azhar.zam, philippe.cattin, \\ georg.rauter) @unibas.ch
}

\begin{abstract}
Typically, Flexoscopes are used in interventions that require minimal number of ports and maneuverability in the target area such as natural orifice transluminal endoscopic surgery. In orthopedic surgeries, these endoscopes are not used because interventions like drilling and osteotomy necessitate large forces. Laser osteotomy does not require mechanical contact with the bone therefore flexible tools can be used for cutting. GG-1 is a custom-designed robotic platform that allows precise control of the flexoscope's pose. The robot is anthropomorphically inspired from a human arm. The robot will be able to move and orient the laser accurately, while following an uneven bone surface in the target area of the osteotomy.
\end{abstract}

\section{Introduction}

Flexible endoscopes (also known as Flexoscopes) are commonly employed in various minimally invasive surgeries (MIS). Typically, Flexoscopes are used in interventions that require minimal number of ports and maneuverability in the target area such as NOTES (natural orifice transluminal endoscopic surgery) (Autorino et al., 2011). In orthopedic surgeries, these endoscopes are not used because interventions like drilling and osteotomy necessitate large forces that can be only exerted by rigid tools (Sugita et al., 2010).

In contrast to traditional orthopedic tools, laser osteotomy does not require mechanical contact with the bone. Consequently, flexible tools can be used for the cutting process (Baek et al., 2015a). Furthermore, the use of lasers is advantageous from the aspects of accuracy and faster healing of the bone (Baek et al., 2015b). Importantly, high accuracy and satisfactory cutting performance of the laser osteotomy can only be reached through stable, dexterous, and precise guidance by a robotic platform.

In this paper, we first present a custom-designed robotic platform that allows precise control of the flexoscope's pose. In the past, flexible endoscopes have been partially automatized by controlling their 
penetration length and orientation, e.g. in colonoscopies (Ruiter et al., 2012). Also passive manipulation of the insertion point and active control of the tip's bending have been realized, e.g. in an endolaryngeal flexible scope (Olds et al., 2012). Another strategy for manipulation in single orifice surgeries uses robotic arms that are extracted from a rigid tube inside the body (Goldman et al., 2013)

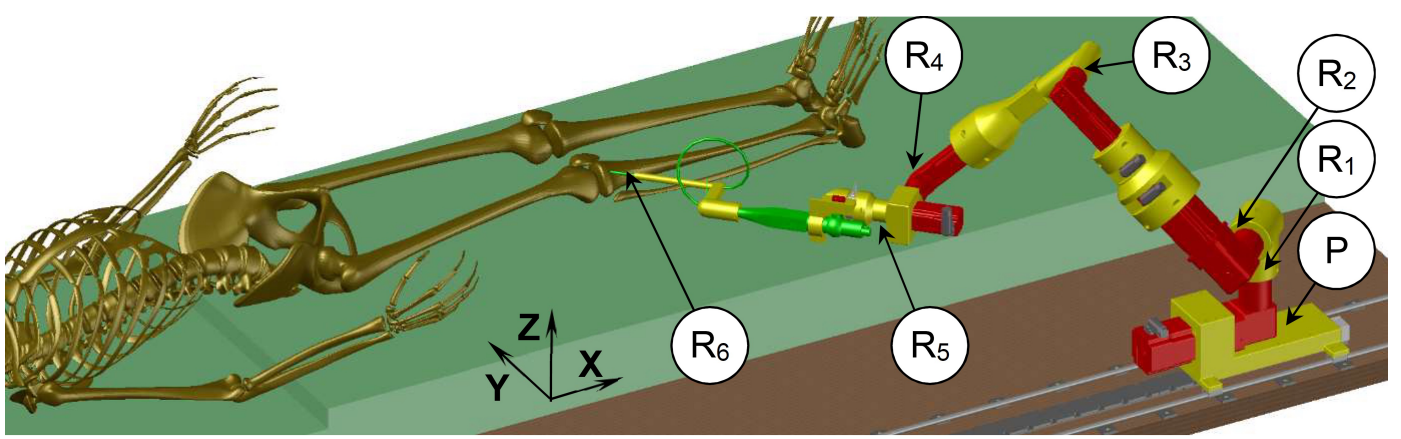

Figure 1: GG-1 approaching the laser osteotomy site on the shinbone. The endoscope is colored light green, the motors are colored red, and the structure yellow.

The herein presented robotic platform uses a serial structure with 7 actuators (see Fig. 1) designed to control the position and orientation of a flexoscope and maneuver it in the body. The robot is anthropomorphically inspired from a human arm in the aspects of link length, joint arrangement and operation method. A special holder was designed to transform the flexible endoscope into a semi rigid endoscope to provide a stable insertion platform for the laser tool enabling only the tip to bend at the end of the holder. The robot will be able to move and orient the laser accurately, while following an uneven bone surface in the target area of the osteotomy.

\section{Materials and Methods}

GG-1, our laser osteotomy robot is a serial robot with a structure of $\mathrm{PR}^{6}$. The first two DoFs (P and $\mathrm{R}_{1}$ ) define a movement in the coronal plane, where the prismatic joint is parallel to the human's longitudinal body axis besides the bed. Next three rotational $\mathrm{DoFs}\left(\mathrm{R}_{2-4}\right)$ determine the insertion point's location and orientation in the coronal plane. The insertion point is used as an RCM (Remote Center of Motion) for intra-body manipulation. The last two joints $\left(\mathrm{R}_{5-6}\right)$ handle the orientation and tip bending of the endoscope that is handled by the robot. Fig. 1 illustrates how GG-1 approaches the knee.

The endoscope currently used in GG-1 is an intubation flexoscope designated 11301BND1 by Karl Storz. The motors used for the joints are: a magnetic linear motor AL2412 for P, two DC servo motors AM8131 with AG2250 WPLE60 perpendicular shaft gearboxes for $\mathrm{R}_{1-2}$, two DC servo motors AM8113 with AG2250 WPLE40 perpendicular shaft gearboxes for $\mathrm{R}_{3-4}$ and an AM8111 motor for $\mathrm{R}_{5}$, all fabricated by Beckhoff Automation $\mathrm{GmbH}$. $\mathrm{R}_{6}$ is driven by a Midi-servo motor 06.SW-0250MG by Savö, rotating the lever of the endoscope. A 6 axial F/T sensor, Mini-45 by ATI, is connected to the base of the endoscope to measure the contact forces of the endoscope with the bone. The links between the joints mainly consist of the electric motors' bodies. 3D printing parts connect the motors to each other and enable a flexible design.

The robot is controlled by an embedded PC CX2020 with a single core Intel i3 processor within a custom designed electric cabinet. Real-time data transfer between motors and sensors is entirely 
handled through the EtherCAT protocol at an update rate of $1 \mathrm{kHz}$. Control is realized through Matlab/Simulink code compiled for TwinCAT3.

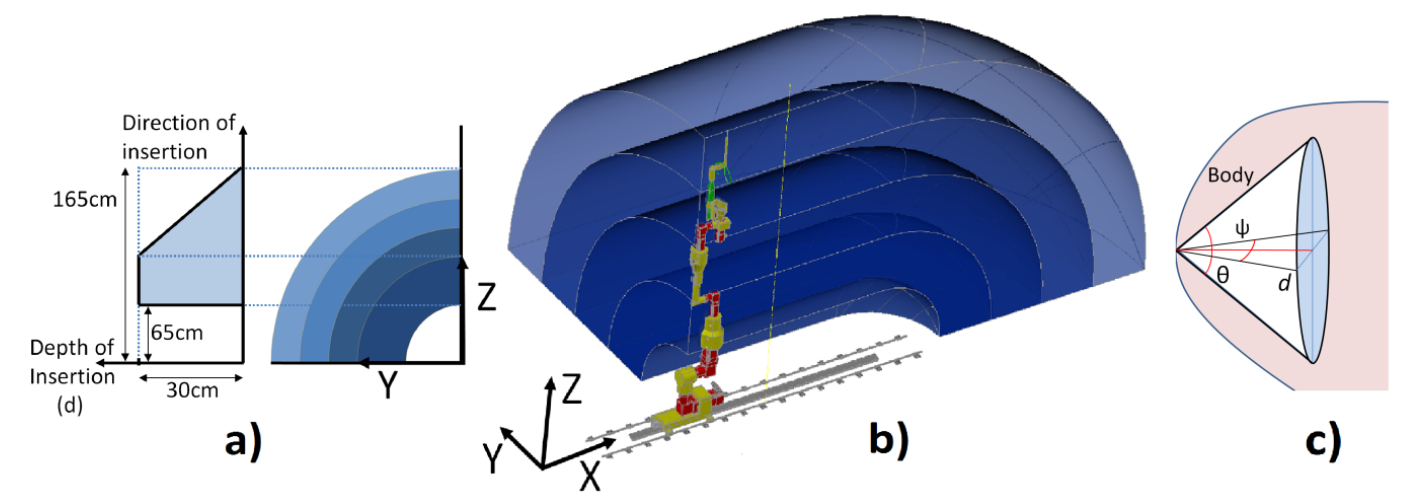

Figure 2: Workspace illustration of GG-1: b) the insertion point's half workspace is illustrated in blue concentric sphere capped cylinders; a) the achievable insertion depth radial to the insertion point workspace illustrated in the $\mathrm{Y}-\mathrm{Z}$ plane, and c) the reachable surgical intra-body volume.

\section{Results}

Based on the dimension of the robot, we calculated its workspace and maneuvering ability in the RCM. The intra-body volume that the system can cover depends on the position of the insertion point as illustrated in Fig. 2. The insertion workspace (the possible positions of an effective post RCM workspace) has the form of a quartered cylinder capped by two one eighth spheres with a radius of 1.6 $\mathrm{m}$, which corresponds to the length of the robot in a fully extended configuration. The post RCM workspace (Fig. 2c) depends on the position and orientation of the insertion point.

The best position to perform the insertion was calculated symbolically and it is located at the midpoint of the workspace illustrated by the second ellipsoid from the bottom in Fig. $2 \mathrm{~b}$ (midpoint of the joint $P$ and $[X, Z]=[0.8,0.8] \mathrm{m}$ from and above $P$; see Fig. 2a-b). The endoscope is able to cover a cone-shaped workspace inside the patient, the angle $\psi$ is bounded to $\pm 45^{\circ}$, the angle $\theta$ to $\pm 90^{\circ}$, and the insertion depth $d$ is between $0-30 \mathrm{~cm}$, which is the total length of the endoscope (Fig. 2c).

\section{Discussion}

In this paper, we present a novel robot for positioning, orienting, and operating a flexible endoscope. The $\mathrm{P}$ joint at the base of the robot enables a large workspace along the operating table and separation of the horizontal and vertical orientation of the robot. This novel bio-inspired design is adapted to the workspace of the surgical arena and tools to fit better to the surgical arena.

The insertion point is defined as an RCM enabling an adequate workspace and a stable frame for the semi flexible endoscope that serves as the end-effector for the laser osteotomy.

It is easy to redesign GG-1 in terms of endoscope fixation, link assembly, to optimally adapt it to different surgical interventions that need more workspace and maneuverability in one or the other direction. 


\section{References}

Autorino R, Cadeddu JA, Desai MM, et al. Laparoendoscopic (2011) Single-site and Natural Orifice Transluminal Endoscopic Surgery in Urology: A Critical Analysis of the Literature. European Urology 59(1), pp: 26-45,

Baek KW, Deibel W, Marinov D, et al. (2015a) Clinical applicability of robot-guided contact-free laser osteotomy in cranio-maxillo-facial surgery: in-vitro simulation and in-vivo surgery in minipig mandibles. British Journal of Oral and Maxillofacial Surgery 53(10), 976-981.

Baek KW, Deibel W, Marinov D, et al.(2015b) A comparative investigation of bone surface after cutting with mechanical tools and Er: YAG laser. Lasers in surgery and medicine 47(5), pp: 426-432, $2015 b$.

Goldman RE, Bajo A, MacLachlan LS, et al. (2013) Design and Performance Evaluation of a Minimally Invasive Telerobotic Platform for Transurethral Surveillance and Intervention. Biomedical Engineering, IEEE Transactions on 60(4), pp: 918-925.

Olds K, Hillel A, Kriss J, et al. (2012) A robotic assistant for trans-oral surgery: the robotic endolaryngeal flexible (Robo-ELF) scope. Journal of Robotic Surgery 6(1), 13-18.

Ruiter J, Rozeboom E, van der Voort M, et al. (2012), Design and evaluation of robotic steering of a flexible endoscope. In Proceedings of 4th IEEE Ras \& Embs International Conference on Biomedical Robotics and Biomechatronics (Biorob), 761-767.

Sugita N, Nakano T, Kato T, et al. (2010), Tool Path Generator for Bone Machining in Minimally Invasive Orthopedic Surgery. IEEE/ASME Transactions on Mechatronics 15(3), 471-479.

\section{Disclosures}

The authors gratefully acknowledge funding of the Werner Siemens Foundation through the MIRACLE project. 